\title{
openheart Prevalence of cardiac pathology and relation to mortality in a multiethnic population hospitalised with COVID-19
}

\author{
Gabriel Bioh, ${ }^{1}$ Christina Botrous, ${ }^{1}$ Emma Howard, ${ }^{1}$ Ashish Patel, ${ }^{1}$ \\ Reinette Hampson, ${ }^{1}$ Roxy Senior (1) ${ }^{1,2,3}$
}

To cite: Bioh G, Botrous C, Howard E, et al. Prevalence of cardiac pathology and relation to mortality in a multiethnic population hospitalised with COVID-19. Open Heart 2021;8:e001833. doi:10.1136/ openhrt-2021-001833

Received 21 August 2021 Accepted 19 October 2021

Check for updates

(c) Author(s) (or their employer(s)) 2021. Re-use permitted under CC BY-NC. No commercial re-use. See rights and permissions. Published by BMJ.

${ }^{1}$ Department of Cardiology, Northwick Park Hospital, Harrow, UK

${ }^{2}$ Royal Brompton Hospital, London, UK

${ }^{3}$ National Heart and Lung Institute, Imperial College London, London, UK

Correspondence to Professor Roxy Senior; roxysenior@cardiac-research. org

\section{ABSTRACT}

Objective To determine the prevalence of cardiac abnormalities and their relationship to markers of myocardial injury and mortality in patients admitted to hospital with COVID-19.

Methods A retrospective and prospective observational study of inpatients referred for transthoracic echocardiography for suspected cardiac pathology due to COVID-19 within a London NHS Trust. Echocardiograms were performed to assess left ventricular (LV), right ventricular (RV) and pulmonary variables along with collection of patient demographics, comorbid conditions, blood biomarkers and outcomes.

Result In the predominant non-white (72\%) population, RV dysfunction was the primary cardiac abnormality noted in $50 \%$ of patients, with RV fractional area change $<35 \%$ being the most common marker of this RV dysfunction. By comparison, LV systolic dysfunction occurred in $18 \%$ of patients. RV dysfunction was associated with LV systolic dysfunction and the presence of a D-shaped LV throughout the cardiac cycle (marker of significant pulmonary artery hypertension). LV systolic dysfunction ( $p=0.002$, HR 3.82, 95\% Cl 1.624 to 8.982), pulmonary valve acceleration time $(p=0.024$, HR 0.98 , $95 \% \mathrm{Cl} 0.964$ to 0.997$)$-marker of increased pulmonary vascular resistance, age $(p=0.047, \mathrm{HR} 1.027,95 \% \mathrm{Cl}$ 1.000 to 1.055) and an episode of tachycardia measured from admission to time of echo ( $p=0.004$, HR $6.183,95 \%$ $\mathrm{Cl} 1.772$ to 21.575 ) were independently associated with mortality.

Conclusions In this predominantly non-white population hospitalised with COVID-19, the most common cardiac pathology was RV dysfunction which is associated with both LV systolic dysfunction and elevated pulmonary artery pressure. The latter two, not RV dysfunction, were associated with mortality.

\section{INTRODUCTION}

During the current COVID-19 pandemic, it has become clear that while initial reports from Wuhan Jin Yin-tan Hospital documented primarily respiratory findings, ${ }^{1}{ }^{2}$ myocardial injury comprises a significant burden of disease, initially indicated by a raised high sensitivity troponin I. $^{1}$ With further noted elevation in N-terminal pro B-type natriuretic

\section{Key questions}

What is already known about this subject?

- Cardiac manifestations of COVID-19 have been apparent from the very beginning of the pandemic.

- Instances of left ventricular (LV) dysfunction due to COVID-19-associated myocarditis and acute coronary syndrome were noted.

- However, it soon emerged that the right ventricle (RV) was predominantly affected but its association with mortality relative to LV dysfunction is conflicting and the aetiology of RV dysfunction is unclear.

What does this study add?

- This study adds to the body of data confirming RV dysfunction as the predominant abnormality in COVID-19 in predominantly non-ventilated patients who are mostly non-white.

- RV dysfunction was associated with pulmonary hypertension and LV dysfunction giving an indication of the aetiology of RV abnormality but LV not RV dysfunction was associated with mortality.

How might this impact on clinical practice?

- The study reinforces the importance of echocardiography use in patients with COVID-19 in highlighting evidence of pulmonary hypertension and LV dysfunction, both adverse prognostic markers which would warrant closer monitoring of patients for clinical deterioration.

peptide (NT-proBNP) of $27.5 \%$ and troponin I of $10 \%$ of a 120 patient sample, the possibility of a COVID-19-related fulminant myocarditis developed in the literature, ${ }^{3}$ reflected by other authors with imaging evidence of severe left ventricular (LV) impairment. ${ }^{4-6}$ Subsequently, right ventricular (RV) dysfunction has also been reported, the aetiology of which can be multifactorial, that is, pulmonary thromboembolic, myocarditis or ischaemic. $^{7-10}$

The cardiac injury, defined by troponin level above the 99th percentile, is thought to occur in $20 \%$ of patients and has been linked to mortality. ${ }^{11}$ Elucidating its aetiology is relevant to understanding the correct 
investigation for, and treatment of patients with, cardiac injury with a view to improving prognosis. While aetiological factors will be multifactorial, to include exacerbation of underlying coronary artery disease or heart failure, metabolic supply demand mismatch due to the infective stress or in fact direct injury from the coronavirus itself, the use of cardiac imaging is crucial to our current understanding. Furthermore, prevalence of cardiac abnormalities and its association with mortality in a multiethnic population hospitalised with COVID-19 is not well demonstrated. It is from this background knowledge that we chose to investigate the prevalence of cardiac abnormalities in the population of patients admitted to hospital with COVID-19 within our trust.

\section{METHODS}

\section{Enrolment}

We prospectively and retrospectively enrolled inpatients within the London North West University Healthcare NHS Trust referred for transthoracic echocardiography for suspected cardiac pathology due to COVID-19 from 1 March to 31 July 2020. All included patients had symptoms suggestive of COVID-19 and either a positive COVID-19 PCR swab or a chest radiograph or a CT scan of the chest demonstrating probable COVID-19, the latter group of patients were clinically managed as patients with COVID19. The data were collected as part of a service evaluation reviewed by Trust R\&D for which individual consent was not required for inclusion of data in the study.

\section{Patient and public involvement}

The emergency circumstances of COVID-19 precluded patient or public involvement in the design, conduct or reporting of this study.

\section{Echocardiography}

Patients had an abbreviated two-dimensional transthoracic echocardiogram study to minimise potential exposure to the COVID-19 virus, based around British Society of Echocardiography guidance. ${ }^{12}$ Measurements were taken as per the American Society of Echocardiography and European Association of Cardiovascular imaging recommendations for cardiac chamber quantification in adults. ${ }^{13} \mathrm{LV}$ function, systolic and diastolic, were assessed. Specific measures of the RV, apart from dimensions, included tricuspid annular plane systolic excursion (TAPSE), RV S velocity on tissue Doppler imaging (RVS'TDI), RV fractional area change (RVFAC) and right ventricular outflow tract velocity time integral (RVOT VTI). Specific measures of pulmonary pressure included pulmonary valve acceleration time (PVAT), estimation of pulmonary artery systolic pressure (PASP) using tricuspid regurgitation to estimate the pressure difference across the tricuspid valve and adding the estimated right atrial pressure (RAP), mean pulmonary artery pressure (mPAP) using peak pulmonary regurgitation velocity to estimate pressure gradient and adding estimated RAP, and pulmonary vascular resistance (PVR) measured using the peak tricuspid regurgitant velocity $(\mathrm{m} / \mathrm{s})$ and dividing this by the RVOT VTI $(\mathrm{cm})$, multiplying the result by 10 and adding a constant 0.16 . Ultrasound enhancing agents were used when images were not diagnostic. The echocardiogram findings were reported and then reviewed by an expert echocardiographer.

Studies were conducted on the Phillips CX50 and Phillips EPIQ CVx ultrasound systems wearing full personal protective equipment. Where ultrasound-enhancing agent was required, LUMINITY (Lantheus medical imaging) was used, given as bolus intravenous injections.

\section{Data collection}

Data recorded for each patient included age, sex, ethnicity, body mass index (BMI), date of symptom onset, comorbid conditions (systemic hypertension, diabetes mellitus, cardiovascular disease, cerebrovascular disease, cancer, chronic kidney disease, respiratory disease), troponin T, NT-proBNP, D-dimer and ferritin. Primary outcome assessed was all-cause mortality.

\section{Statistical analysis}

The IBM SPSS statistics V.27 package was used for all statistical analysis. In all analyses, $p$ values of less than 0.05 were considered statistically significant. Normally distributed continuous data were analysed using the Student's t-test with normality assessed using quantile-quantile plots and the Shapiro-Wilk test. Non-normally distributed continuous data were analysed using the Mann-Whitney $\mathrm{U}$ test.

Categorical data were analysed using the $\chi^{2}$ test of independence; however, where expected frequencies were less than five in $20 \%$ or more of cells, or sample size was less than 40, Fisher's exact test was used for analysis.

Logistic regression was used to determine predictors of abnormal RV function. Following univariable analysis and collinearity diagnostics (using a cut-off for variance inflation factor of $>5$, condition index $>15$ and variance proportions $>0.5$ ), only significant variables were entered into a multivariable analysis using the backward elimination method.

Kaplan-Meier survival analysis with log rank test were used to analyse time to death. Cox proportional hazards model was used to determine the hazard of death for multiple covariates and their interaction. To test the assumption of constant hazard ratio (HR) over time, we used visual assessment of $\log (-\log ($ survival $))$ plots. Following univariable analysis, significant variables were entered into the multivariable analysis using the backward elimination method.

\section{RESULTS}

\section{Patient demography}

In total, we enrolled 120 patients referred for a clinically indicated echocardiogram. Forty-two had their echocardiogram on the intensive therapy unit (ITU) and 78 on the general ward. Table 1 shows the patient demography. The average age of patients was 66.9 years, $67 \%$ were 
Table 1 Recorded variables for demographics, blood work, comorbid conditions, haemodynamics and ventilation with means and SDs or medians and IQRs for continuous variables and number and percentage for categorical variables

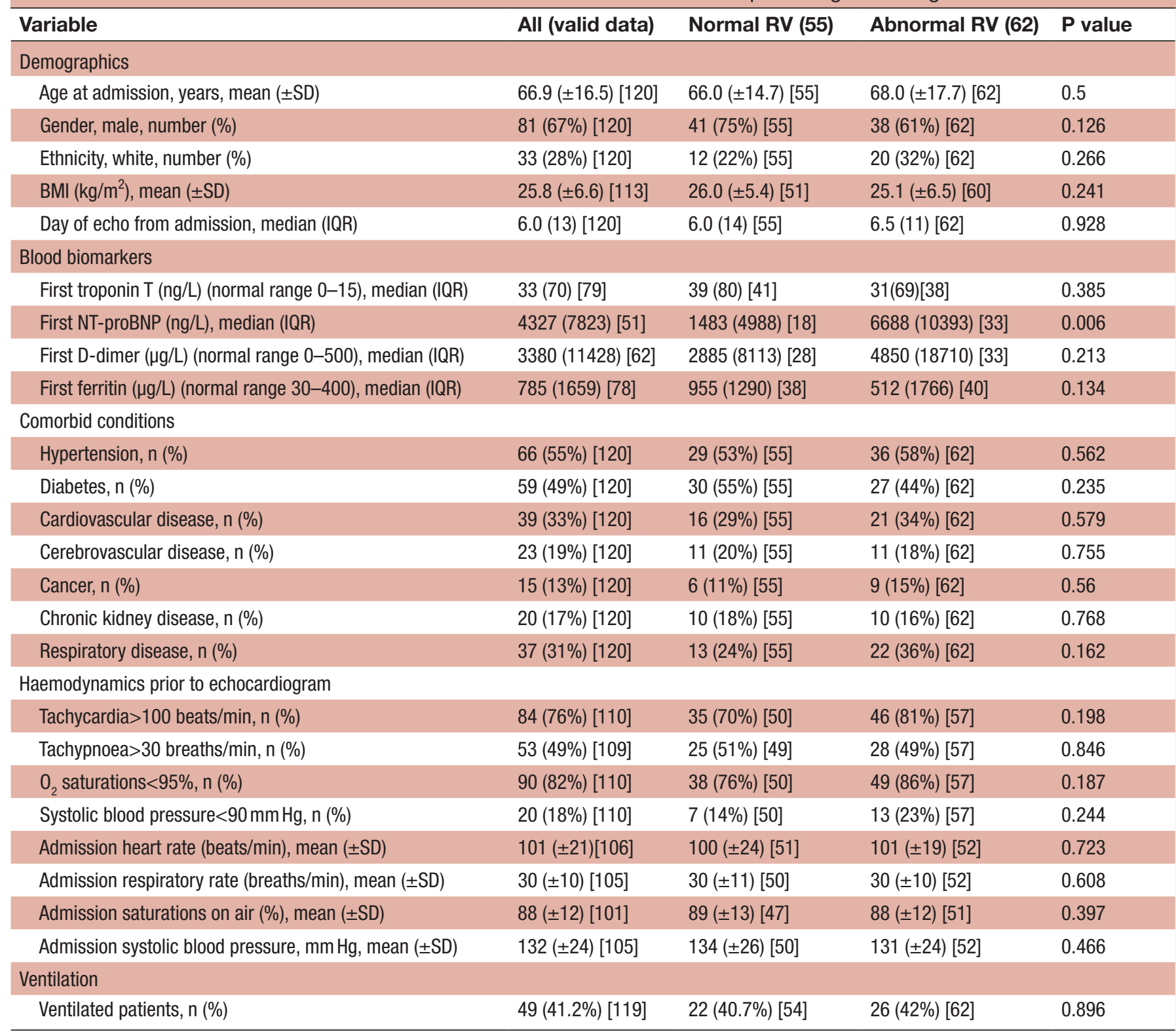

Number in square bracket records total number of patients.

$\mathrm{BMI}$, body mass index; NT-proBNP, N-terminal pro B-type natriuretic peptide; RV, right ventricular.

male, $28 \%$ defined ethnicity as white and the average BMI was $25.8 \mathrm{~kg} / \mathrm{m}^{2}$. Of comorbid conditions, systemic hypertension was the most common, present in $55 \%$ of patients, followed by diabetes mellitus, present in $49 \%$ and then cardiovascular disease (known ischaemic heart disease, cardiomyopathy, significant valvulopathy or arrhythmia) in $33 \%$ of patients. Other comorbid conditions are recorded in table 1.

\section{Echocardiographic features}

Echocardiographic abnormalities were broadly categorised into LV, RV and pulmonary artery pressure measures (table 2). Of the 119 patients with echocardiographic data (1 patient with very poor windows due to grossly elevated BMI was removed from echocardiographic assessment), 27 (23\%) demonstrated abnormal LV systolic function $(\mathrm{EF}<50 \%), 16(17 \%)$ of 93 patients (in whom LV filling pressure was recorded) had elevated LV filling pressure with normal LV ejection fraction (LVEF). Right ventricular impairment by any measure (Visual, RVFAC, TAPSE, RVS'TDI) was present in $62(53 \%)$ of 117 patients and $42 \%$ of patients showed RV dilatation. The most common measure of RV impairment was fractional area change (FAC) of $<35 \%$, present in 47 (53\%) of 87 patients. This was followed by TAPSE $<1.7$, abnormal in $17(39 \%)$ of 44 patients and then RVS'TDI, reduced in 22 (20\%) of 110 patients. LV regional wall motion abnormality (RWMA) was noted in $14(12 \%)$ patients. 
Table 2 Recorded variables for left ventricle, right ventricle and pulmonary pressure with means and SDs for continuous variables and number and percentage for categorical variables

\begin{tabular}{|c|c|c|c|c|}
\hline Variable & All (valid data) & Normal RV (55) & Abnormal RV (62) & $P$ value \\
\hline \multicolumn{5}{|l|}{ Left ventricle } \\
\hline New LV systolic dysfunction, $\mathrm{n}(\%)$ & $22(18 \%)[119]$ & $6(11 \%)[55]$ & $16(26 \%)[62]$ & 0.04 \\
\hline LV end-diastolic dimension (cm), mean ( \pm SD) & $4.5( \pm 0.7)[117]$ & $4.4( \pm 0.6)[54]$ & $4.6( \pm 0.8)[61]$ & 0.06 \\
\hline LA vol index $\left(\mathrm{mL} / \mathrm{m}^{2}\right)$, mean $( \pm \mathrm{SD})$ & $34.8( \pm 17.9)[100]$ & $31.4( \pm 14.8)[46]$ & $37.6( \pm 19.8)[54]$ & 0.074 \\
\hline $\mathrm{E} / \mathrm{A}$, mean $( \pm \mathrm{SD})$ & $1.0( \pm 0.4)[93]$ & $0.95( \pm 0.4)[47]$ & $1.0( \pm 0.4)[44]$ & 0.336 \\
\hline $\mathrm{E} / \mathrm{E}^{\prime}$ average, mean $( \pm \mathrm{SD})$ & $12.7( \pm 6.8)[106]$ & $12.4( \pm 6.7)[51]$ & $13.2( \pm 6.9)[53]$ & 0.459 \\
\hline$E / E^{\prime}$ average $>14, n(\%)$ & $35(33 \%)[106]$ & $16(31 \%)[51]$ & $19(36 \%)[53]$ & 0.629 \\
\hline New elevated LV filling pressure+normal LVEF $>50 \%, n(\%)$ & $7(8 \%)[93]$ & $5(11 \%)[44]$ & $2(6 \%)[49]$ & 0.249 \\
\hline New LV dysfunction (systolic or diastolic), $\mathrm{n}(\%)$ & $29(24 \%)[119]$ & $11(20 \%)[55]$ & $18(29 \%)[62]$ & 0.259 \\
\hline TAPSE $(\mathrm{cm})$, mean $( \pm \mathrm{SD})$ & $2.5( \pm 3.4)[44]$ & $2.3( \pm 0.4)[16]$ & $1.5( \pm 0.4)[27]$ & $<0.001$ \\
\hline Reduced TAPSE $<1.7 \mathrm{~cm}, \mathrm{n}(\%)$ & $17(39 \%)[44]$ & $1(6 \%)[16]$ & $16(59 \%)[27]$ & 0.001 \\
\hline RVS'TDI (cm/s), mean $( \pm S D)$ & $13.2( \pm 4.2)[110]$ & $15.2( \pm 3.5)[50]$ & $11.5( \pm 4.2)[59]$ & $<0.001$ \\
\hline Reduced RVS'TDI<9.5cm, n (\%) & $22(20 \%)[110]$ & $0(0 \%)[50]$ & $22(37 \%)[59]$ & $<0.001$ \\
\hline FAC $(\%)$, mean $( \pm S D)$ & $34.5( \pm 12.2)[87]$ & $45.6( \pm 7.5)[35]$ & $27.1( \pm 8.4)[52]$ & $<0.001$ \\
\hline Reduced $\mathrm{FAC}<35 \%, \mathrm{n}(\%)$ & $47(53 \%)[88]$ & $0(0 \%)[35]$ & $47(90 \%)[52]$ & $<0.001$ \\
\hline RV dilatation, n (\%) & $43(42.2 \%)[102]$ & $9(20 \%)[45]$ & $34(60 \%)[57]$ & $<0.001$ \\
\hline Basal RV diameter $(\mathrm{cm})$, mean $( \pm \mathrm{SD})$ & $4.0( \pm 0.8)[102]$ & $3.7( \pm 0.6)[45]$ & $4.2( \pm 0.8)[57]$ & 0.001 \\
\hline D-shaped LV, n (\%) & $25(21 \%)[119]$ & $1(2 \%)[55]$ & $24(39 \%)[62]$ & $<0.001$ \\
\hline PV acceleration time $(\mathrm{ms})$, mean $( \pm \mathrm{SD})$ & $97.0( \pm 27)[97]$ & $107.5( \pm 25.6)[38]$ & $90.9( \pm 26.5)[57]$ & 0.003 \\
\hline PVAT<80 ms, n (\%) & $29(30 \%)[97]$ & $7(18 \%)[38]$ & $21(37 \%)[57]$ & 0.054 \\
\hline TR velocity $\mathrm{m} / \mathrm{s}$, mean $( \pm \mathrm{SD})$ & $2.8( \pm 0.6)[80]$ & $2.7( \pm 0.5)[31]$ & $2.9( \pm 0.6)[49]$ & 0.039 \\
\hline Pulmonary artery systolic pressure $(\mathrm{mm} \mathrm{Hg})$, mean $( \pm \mathrm{SD})$ & $40.5( \pm 16.2)[81]$ & $33.3( \pm 9.3)[32]$ & $45.2( \pm 18.0)[49]$ & 0.003 \\
\hline $\mathrm{PASP}>50 \mathrm{~mm} \mathrm{Hg}, \mathrm{n}(\%)$ & $21(26 \%)[81]$ & $3(9 \%)[32]$ & $18(37 \%)[49]$ & 0.006 \\
\hline Pulmonary vascular resistance (Wood units), mean $( \pm S D)$ & $2.8( \pm 1.4)[72]$ & $2.0( \pm 0.6)[27]$ & $3.3( \pm 1.5)[45]$ & $<0.001$ \\
\hline PVR>3.0, n (\%) & $25(35 \%)[72]$ & $2(7 \%)[27]$ & $23(51 \%)[45]$ & $<0.001$ \\
\hline Elevated pulmonary pressure, $\mathrm{n}(\%)$ & $47(47 \%)[100]$ & $12(29 \%)[41]$ & $35(60 \%)[58]$ & 0.002 \\
\hline RA filling pressure $(\mathrm{mm} \mathrm{Hg})$, mean $( \pm \mathrm{SD})$ & $7.6( \pm 6.0)[94]$ & $4.2( \pm 2.9)[44]$ & $10.6( \pm 6.6)[58]$ & $<0.001$ \\
\hline
\end{tabular}

Number in square bracket records total number of patients.

LV, left ventricular; LVEF, LV ejection fraction; PASP, pulmonary artery systolic pressure; PVR, pulmonary vascular resistance; RVOT VTI, right ventricular outflow tract velocity time integral; RWMA, regional wall motion abnormality; TAPSE, tricuspid annular plane systolic excursion.

Review of historic echocardiograms revealed that 5 of the 27 patients with LV systolic dysfunction had similar previously documented LV systolic dysfunction, 8 of the 16 patients had previously documented elevated LV filling pressure, 8 of the 14 patients had RWMA previously noted and 4 of the 62 patients had previously documented RV dysfunction. As a result of this, pathology due to COVID-19 is more accurately determined to cause LV 
systolic dysfunction in 22 (18\%) of 119 patients, elevated LV filling pressure in $8(9 \%)$ of 93 patients, RWMA in 6 $(5 \%)$ of the 119 patients and RV dysfunction in 58 (50\%) of 117 patients.

Assessment of significant pulmonary pressure elevation by means of PVAT $<80 \mathrm{~ms}$ or PASP $>50 \mathrm{mmHg}$ or PVR $>3$ Woods units (WU) resulted in $47(47 \%)$ out of 100 patients having significantly elevated pulmonary pressures. Considering the individual variables, PVR was $>3.0$ in $25(35 \%)$ of 72 patients, PVAT was $<80 \mathrm{~ms}$ in $29(30 \%)$ of 97 patients and PASP $>50 \mathrm{~mm} \mathrm{Hg}$ in 21 (26\%) of 81 patients. Visual evaluation of elevated RV pressure through a D-shaped LV throughout the cardiac cycle was present in 25 (21\%) of 119 patients.

Considering RV dysfunction to be the predominant cardiac abnormality, we analysed all variables split into normal (55 patients) versus abnormal (62 patients) RV function by any measure (tables 1 and 2). No significant difference was found with regard to demographics or comorbid conditions. NT-proBNP, however, was significant with a mean of $6024 \mathrm{ng} / \mathrm{L}$ (median $1483 \mathrm{ng} / \mathrm{L}$ ) versus $11273 \mathrm{ng} / \mathrm{L}$ (median $6688 \mathrm{ng} / \mathrm{L}$ ) in normal versus abnormal RV function respectively, $\mathrm{p}=0.006$. LV systolic dysfunction and LV end-systolic dimension were significantly increased in patients with abnormal RV function, $\mathrm{p}=0.04$ and $\mathrm{p}=0.014$, respectively.

Of pulmonary variables, PVAT was significantly lower in the abnormal (mean $90.9 \mathrm{~ms}$ ) compared with normal (mean $107.5 \mathrm{~ms}$ ) RV function group. Tricuspid regurgitation velocity, PASP, PVR and elevated pulmonary pressure by any measure (PVAT $<80 \mathrm{~ms}$ or PASP $\geq 50 \mathrm{~mm} \mathrm{Hg}$ or PVR $>3 \mathrm{WU}$ ) were all significantly higher in patients with abnormal RV function.

Haemodynamic and respiratory data were recorded for 110 patients prior to echocardiogram with at least 1 episode of desaturation to below $95 \%$ found in $82 \%$ of patients, tachycardia ( $>100$ beats $/ \mathrm{min})$ in $76 \%$ of patients, tachypnoea over 30 breaths/min in $59 \%$ of patients and systolic blood pressure less than $90 \mathrm{~mm} \mathrm{Hg}$ in $18 \%$ of patients. There was no significant difference between the normal and abnormal RV patients when using these cut-offs or the admission haemodynamic and respiratory data.

\section{Association of variables with RV dysfunction}

Logistic regression analysis was conducted for the assessment of association with RV dysfunction by any measure. Univariable analysis included demographics, blood biomarkers, comorbid conditions, cardiac enzymes, haemodynamics, LV variables and pulmonary artery variables. This identified LV systolic dysfunction, LV endsystolic dimension, D-shaped LV, PVAT, TR Velocity, PASP, PASP $>50 \mathrm{~mm} \mathrm{Hg}, \mathrm{PVR}$ and $\mathrm{PVR}>3.0$ as significant variables associated with abnormal RV function. Following collinearity diagnostics, multicollinearity was established between TR velocity, PASP and PASP $>50$. PASP was chosen for input into multivariable analysis. Given LV end-systolic dimension is a measure of LV systolic function it was not entered into the multivariable analysis. Finally, an interaction between PVR and D-shaped LV was discovered when both were input into the multivariable analysis. D-shaped LV was chosen as there was no missing data compared with missing PVR measures in 45 of the patients. Multivariable analysis determined only LV systolic dysfunction and D-shaped LV to be significantly associated with abnormal RV function (table 3).

\section{Outcome of patients}

Patients were enrolled and followed up over a study period of 5 months with 51-day follow-up of the last patient enrolled by the end of the study period. During follow-up, there were 33 deaths. Kaplan-Meier survival analysis showed no significant difference between normal and abnormal RV function, $\mathrm{p}=0.39$. However, of individual markers of RV function, abnormal TAPSE was significant for reduced survival $\mathrm{p}=0.038$ (figure 1 ). Of measures of pulmonary pressure, PVAT $<80 \mathrm{~ms}$ was significant for reduced survival $p=0.01$, but when the composite of any measure of pulmonary pressure elevation was taken, it was not significant (figure 2). There was

Table 3 Significant $(\mathrm{p}<0.05)$ predictor variables for RV dysfunction using logistic regression

\begin{tabular}{|c|c|c|}
\hline Variable & Univariable & Multivariable \\
\hline LV end-systolic dimension (cm) & OR $2.34(\mathrm{Cl} 1.32$ to 4.14$) \mathrm{p}=0.003$ & \\
\hline PV acceleration time (ms) & OR $0.98(\mathrm{Cl} 0.96$ to 1.00$) \mathrm{p}=0.005$ & \\
\hline TR Velocity (m/s) & OR $2.42(\mathrm{Cl} 1.02$ to 5.78$) p=0.046$ & \\
\hline $\mathrm{PASP}>50 \mathrm{~mm} \mathrm{Hg}$ & OR $5.61(\mathrm{Cl} 1.50$ to 21.07$) \mathrm{p}=0.011$ & \\
\hline Pulmonary vascular resistance (Wood units) & OR $3.53(\mathrm{Cl} 1.72$ to 7.23$) \mathrm{p}=0.001$ & \\
\hline$P V R>3.0$ & OR $13.07(\mathrm{Cl} 2.76$ to 61.84$) \mathrm{p}=0.001$ & \\
\hline
\end{tabular}

Univariable analysis results are given as $\mathrm{OR}$ with $95 \% \mathrm{Cl}$ and $\mathrm{p}$ value. After testing for collinearity of the predictor variables with $\mathrm{p}<0.05$, multivariable analysis was conducted with significant variables recorded giving $\mathrm{OR}, 95 \% \mathrm{Cl}$ and $\mathrm{p}$ value. LV, left ventricular; PASP, pulmonary artery systolic pressure; PVR, pulmonary vascular resistance. 


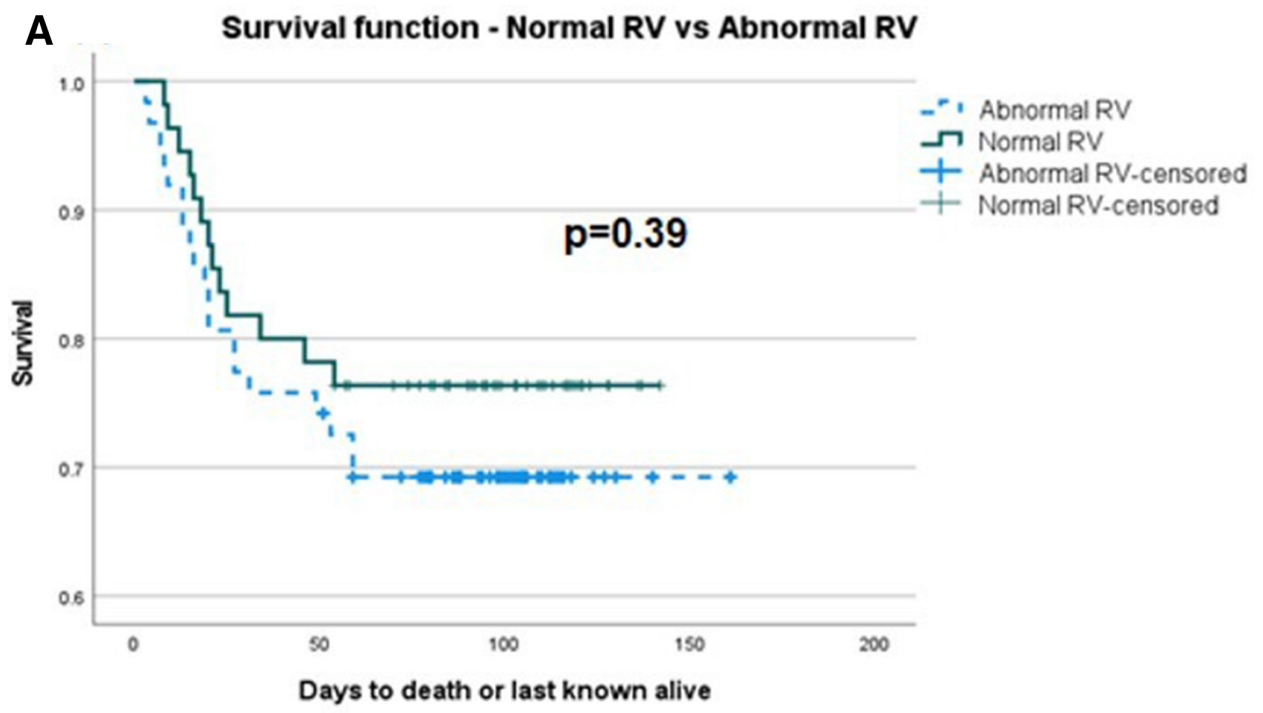

B Survival Function- TAPSE $\geq 1.7$ vS TAPSE $<1.7$

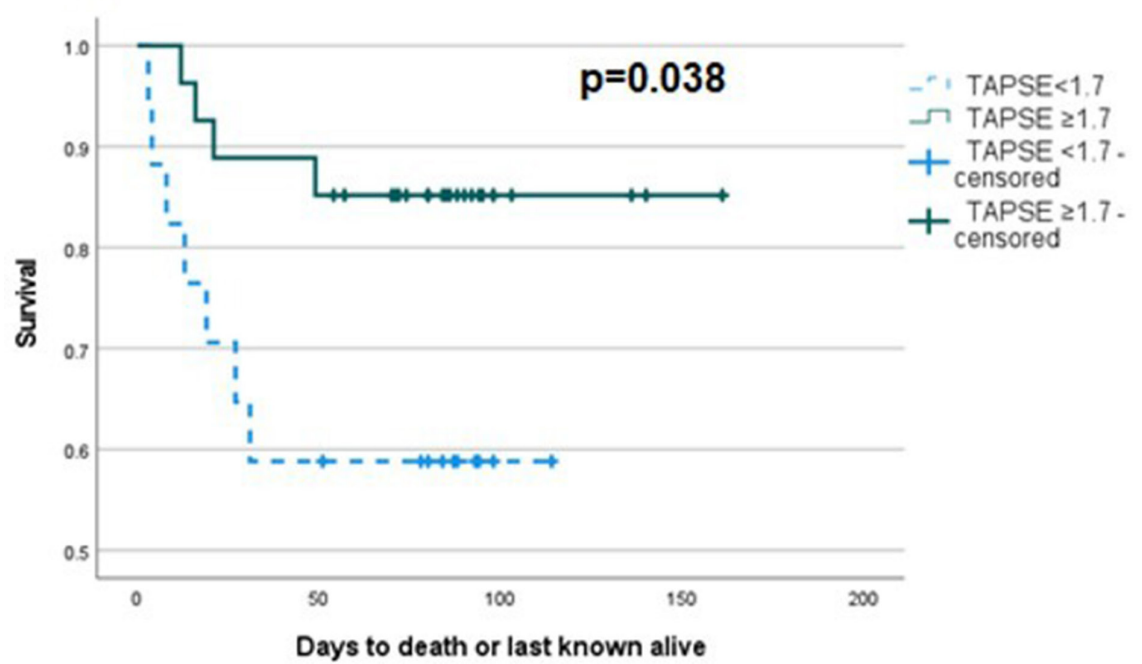

Figure 1 Kaplan-Meier survival curve of (A) normal and abnormal RV function, log rank $p=0.39$, (B) normal and abnormal TAPSE, log rank $\mathrm{p}=0.038$. RV, right ventricular; TAPSE, tricuspid annular plane systolic excursion.

a significant difference between normal and abnormal LV systolic function, $p=0.03$. Considering the haemodynamic and respiratory data, only an episode of tachycardia from admission to the time of echocardiogram demonstrated a significant difference in survival, $p=0.03$ (figure 3). Of interest, Kaplan-Meier survival analysis showed a reduced time to mortality in patients with RWMA but missed statistical significance, $\mathrm{p}=0.051$.

Among patient demography, comorbid conditions, cardiac biomarkers, haemodynamics, LV, RV and pulmonary artery assessment parameters, we found age, LV systolic dysfunction, tachycardia, TAPSE, PVAT and PVR were significant on univariable analysis using Cox proportional hazards model for the outcome of mortality. Using age, LV systolic dysfunction, tachycardia and PVAT (TAPSE and PVR had most missing values) for multivariable analysis, all were significant predictors of mortality, age ( $\mathrm{p}=0.047$, HR $1.027,95 \%$ CI 1.000 to 1.055$)$, PVAT
( $\mathrm{p}=0.036$, HR 0.981 , CI 0.964 to 0.999$)$, LV systolic dysfunction ( $\mathrm{p}=0.002$, HR 4.0351, CI 1.683 to 9.676) and tachycardia ( $\mathrm{p}=0.004$, HR 6.469 , CI 1.841 to 22.735 ).

\section{DISCUSSION}

In this study, we assessed the prevalence and type of cardiac abnormalities and their association with mortality in consecutive patients admitted to the hospital with symptoms suggestive of COVID-19 and PCR confirmed COVID-19 or clinical features and chest radiograph or CT chest suggestive of the infection in a patient population which is predominantly (almost 75\%) non-white. The data showed a new echocardiographic abnormality related to LV dysfunction was seen in $24 \%$ of patients, of which LV systolic dysfunction occurred in $18 \%$ of patients and elevated LV filling pressure with LVEF $>50 \%$ in $8 \%$ of patients. New RWMA was present in only $5 \%$ of patients. 
A

Survival Function- PVAT $>80 \mathrm{~ms}$ vs PVAT $\leq 80 \mathrm{~ms}$

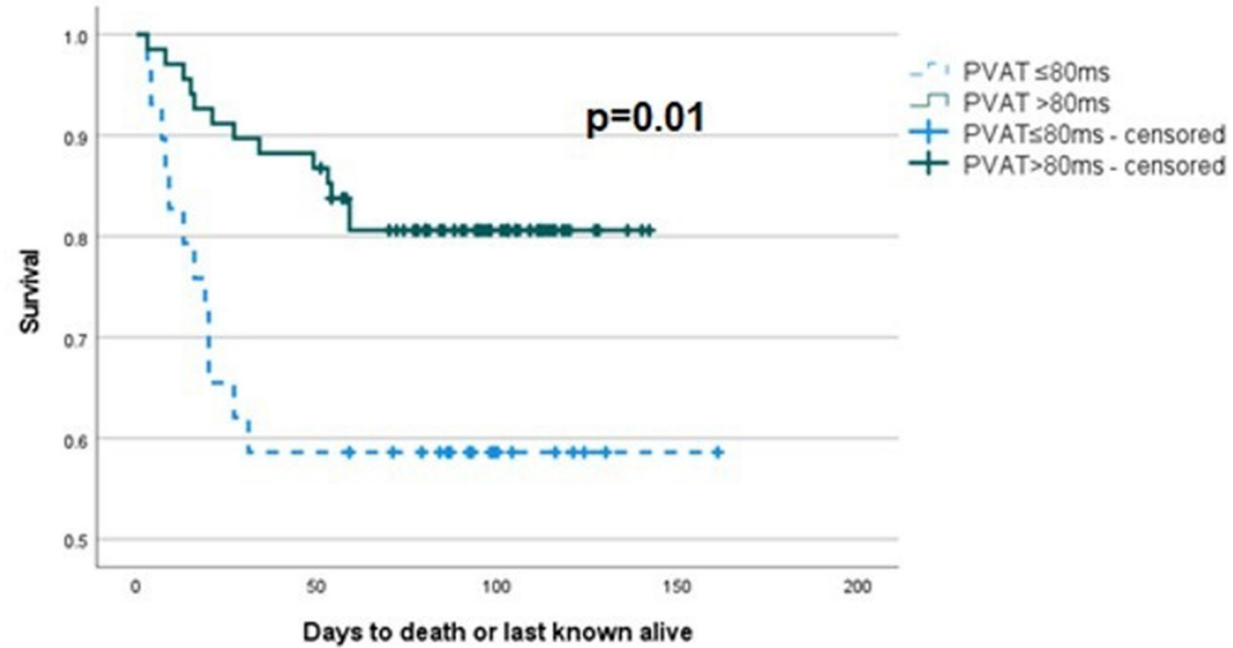

B

\section{Survival Function-Elevated vs Normal pumonary pressure by any measure}

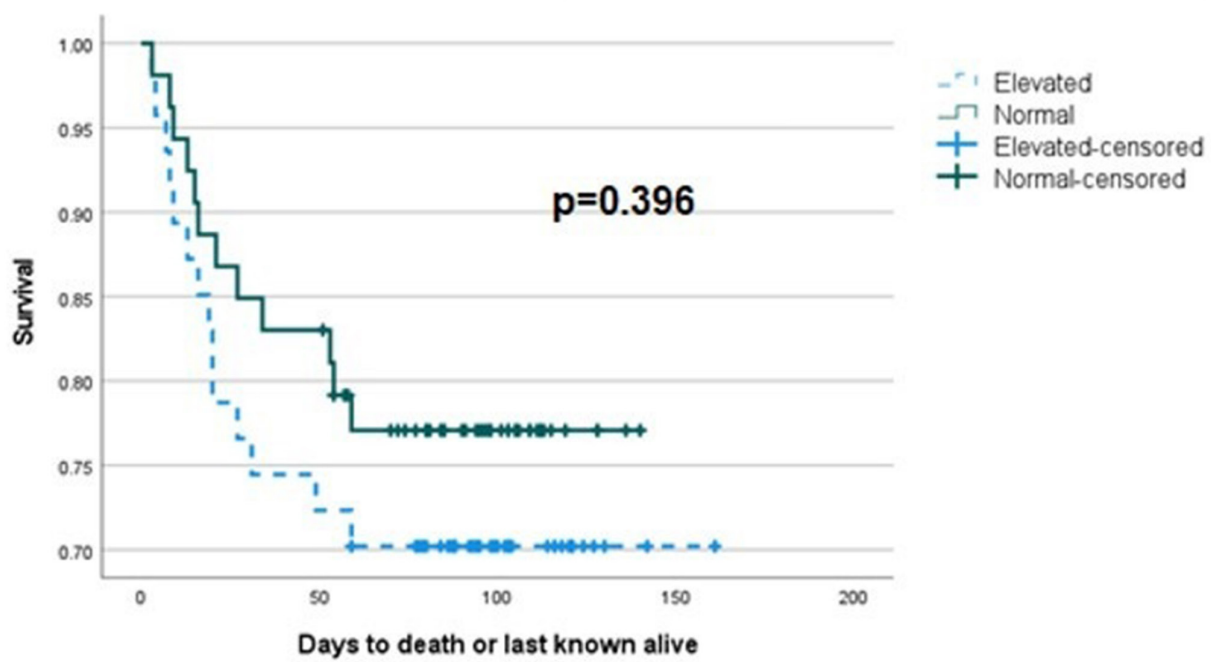

Figure 2 Kaplan-Meier survival curve of (A) PVAT $>80 \mathrm{~ms}$ vs PVAT $\leq 80 \mathrm{~ms}$, log rank $\mathrm{p}=0.01$, (B) elevated and normal pulmonary pressure by any measure, log rank $\mathrm{p}=0.396$. PVAT, pulmonary valve acceleration time.

Fifty per cent of the patients demonstrated RV dysfunction. The independent factors associated with RV dysfunction were LV systolic dysfunction and pulmonary artery hypertension. Furthermore, age, LV systolic dysfunction, pulmonary artery hypertension and tachycardia were independently associated with all-cause mortality.

The predominant cardiac abnormality, in this study, was RV dysfunction. This has been shown previously but our study demonstrated this in a predominantly nonwhite population suggesting that the predominant RV dysfunction is likely to be independent of white versus non-white population. When considering the aetiology of RV dysfunction, the above data demonstrated an increase in pulmonary pressures in $47 \%$ of patients, suggesting it may be responsible for part of the RV disease burden. The test of biological plausibility is met through an established primary lung insult caused by COVID-19 and a COVID-19-associated acute respiratory distress syndrome
(ARDS), ${ }^{1415}$ as well as right heart disease secondary to classical ARDS. ${ }^{16}$ Further clues to the mechanism of RV dysfunction secondary to the primary lung disease may be derived from the nature of dysfunction. Significantly more patients demonstrated radial (53\%) as opposed to longitudinal (20\%) dysfunction. The relative sparing of RV longitudinal function with primary radial dysfunction was also observed in another study. ${ }^{9}$ The echocardiographic appearance is somewhat similar to McConnell's sign in acute pulmonary embolism (PE) which results in acute increase of pulmonary artery pressure causing afterload mismatch and acute RV dysfunction with akinesia of the mid RV free wall but hyperkinesia of the apical RV free wall.

High PASP $(>50 \mathrm{~mm} \mathrm{Hg})$ and PVR $(>3 \mathrm{WU})$ were observed in more than half of the patients with RV dysfunction and a feature of high pulmonary artery pressure, D-shaped LV in systole, was independently associated 


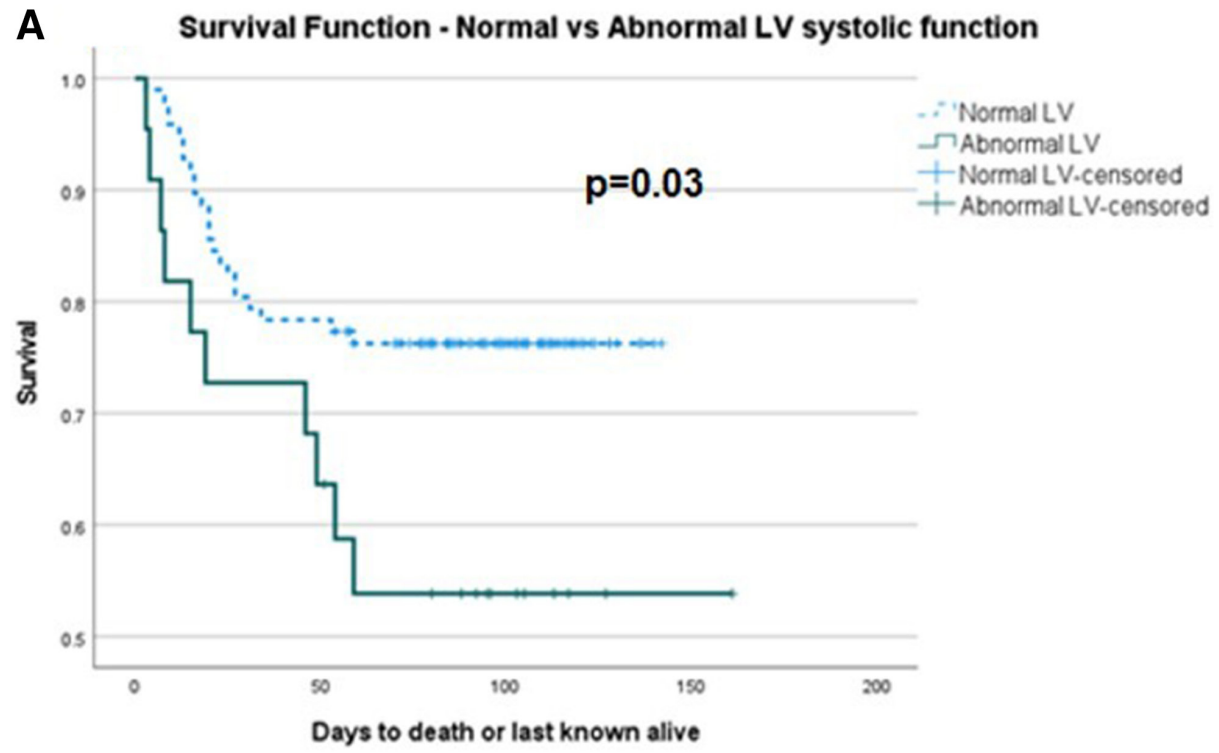

B Survival Function- Episode of tachycardia vs No episode of tachycardia

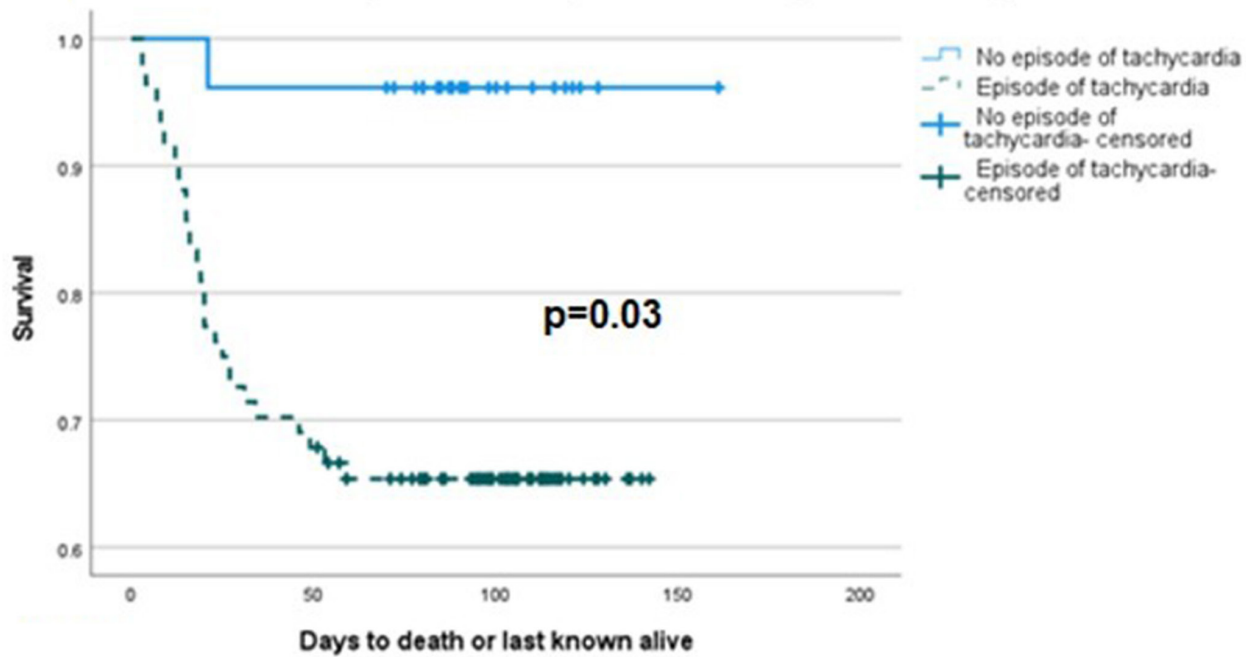

Figure 3 Kaplan-Meier survival curve of (A) normal and abnormal LV systolic function, log rank $p=0.03$, (B) episode of tachycardia from admission to date of echocardiogram and no episode of tachycardia from admission to date of echocardiogram, log rank $\mathrm{p}=0.03$. LV, left ventricular.

with RV dysfunction. As such, a potential mechanism of subsegmental PE or in situ thrombus provoked by SARSCoV-2 must also be considered. Added to this, RV distension under pressure is reflected in raised NT-proBNP, significantly higher in patients with abnormal RV function compared with normal RV function. LV systolic dysfunction, which was also independently associated with RV dysfunction, could also have contributed to the occurrence of pulmonary hypertension, although features of raised LV filling pressure were not associated with RV dysfunction.

However, we must also consider that there were patients with abnormal RV function but normal pulmonary pressures and vice versa. Thus, other causes including primary direct viral damage to the RV myocardium through myocarditis need to be considered. Such a mechanism would most likely also be responsible for the left ventricular dysfunction seen in COVID-19 which is predominantly global as opposed to regional. Almost $20 \%$ of patients demonstrated likely new LV systolic dysfunction secondary to COVID-19 and LV systolic dysfunction was independently associated with RV dysfunction but not raised LV filling pressure. This would support a hypothesis of a myocarditic mechanism and primary elevated pulmonary pressures as causes for RV dysfunction, respectively.

The independent associations with mortality in this study were LV systolic dysfunction, pulmonary artery hypertension and tachycardia. If it is considered that such patients with LV systolic dysfunction secondary to COVID-19 have an underlying myocarditic process, we could conclude that myocarditis is a key cause of mortality in COVID-19. Abnormal TAPSE, although not independently associated with mortality, was a univariable associate but the more common abnormality of radial function was not associated with mortality. The latter abnormality was 
more commonly associated with acute pulmonary hypertension in this study. Thus, it is possible that the abnormal TAPSE reflects a myocarditic process and so predicts mortality in the same way as LV systolic dysfunction. Alternatively, it is possible that the pulmonary pressure when severely elevated also affects RV longitudinal function as well as radial and therefore predicts mortality due to severe elevation of pulmonary pressure which is reflected in the fact that PVAT $\leq 80 \mathrm{~ms}$ - a measure of pulmonary vascular resistance-confers reduced survival in patients with COVID-19. Tachycardia is a reflection of significant global cardiac disease and systemic inflammation.

\section{LIMITATIONS}

The data presented are from a single centre and therefore may be subject to confounders specific to the locality, for example, age, ethnicity, social class, and therefore not representative of the general population. Specifically, the demographic data recorded a $72 \%$ non-white population. While having data from such a diverse population is itself of interest, the data may have limited application to the broader white population in the UK. Added to this, the relatively small numbers limit the ability to determine prevalence of cardiac abnormalities accurately within the population.

As a retrospective and prospective observational study, it will be subject to selection biases which cannot be controlled for. Such biases include the clinical criteria for patients requiring an echocardiogram which was not standardised but would vary between clinical teams and could possibly have evolved as the pandemic evolved and we learnt more about the natural history of the disease.

\section{CONCLUSION}

On the basis of the present data, it appears that RV dysfunction is a predominant pathology due to pulmonary hypertension and LV systolic dysfunction. Thrombotic or thromboembolic pulmonary disease may be the primary cause of RV dysfunction. However, LV dysfunction appears to be predominantly primary and likely myocarditic in aetiology. Both LV systolic dysfunction and pulmonary hypertension appear to be the major driver of mortality. Such data can only be considered hypothesis generating. Thus, larger studies, meta-analysis, imaging studies of other modalities and histopathological studies are required to shed more light on the aetiology of the cardiac manifestations of COVID-19 and association with mortality.

Contributors RS and GB conceived of the presented idea. GB and EM designed and implemented the study. GB, CB and AP performed imaging for the study. GB, $\mathrm{EM}, \mathrm{CB}, \mathrm{AP}$ and $\mathrm{RH}$ performed data collection. $\mathrm{GB}$ analysed and reported the data under the supervision of RS. All authors discussed the results and contributed to the final manuscript. GB and RS were responsible for the overall content of the manuscript. RS is the guarantor.
Funding The study received funding from Northwick Park Cardiac Research Charity. The funder had no involvement in the study design, collection, analysis and interpretation of the data, writing of and decision to submit the paper for publication.

Competing interests Professor Senior was given speaker fees by Bracco, Milan, Italy, Lantheus Medical Imaging, Boston, Massachusetts and Philips Healthcare, Eindhoven, Holland.

Patient consent for publication Not applicable.

Ethics approval The data were collected as part of a service evaluation reviewed and approved by the London North West University Healthcare NHS Trust R\&D Department in April 2020, evaluation number SE20/001. As this was a service evaluation, ethics committee approval was not required.

Provenance and peer review Not commissioned; internally peer reviewed.

Data availability statement Data are available upon reasonable request. The final anonymous dataset that supports the findings of this study are available from the corresponding author, $\mathrm{RS}$, on reasonable request.

Open access This is an open access article distributed in accordance with the Creative Commons Attribution Non Commercial (CC BY-NC 4.0) license, which permits others to distribute, remix, adapt, build upon this work non-commercially, and license their derivative works on different terms, provided the original work is properly cited, appropriate credit is given, any changes made indicated, and the use is non-commercial. See: http://creativecommons.org/licenses/by-nc/4.0/.

ORCID iD

Roxy Senior http://orcid.org/0000-0001-6579-7039

\section{REFERENCES}

1 Huang C, Wang Y, Li X, et al. Clinical features of patients infected with 2019 novel coronavirus in Wuhan, China. Lancet 2020;395:497-506.

2 Chen N, Zhou M, Dong X, et al. Epidemiological and clinical characteristics of 99 cases of 2019 novel coronavirus pneumonia in Wuhan, China: a descriptive study. Lancet 2020;395:507-13.

3 Chen C, Zhou Y, Wang DW. SARS-CoV-2: a potential novel etiology of fulminant myocarditis. Herz 2020;45:230-2.

4 Ruan Q, Yang K, Wang W. Clinical predictors of mortality due to COVID-19 based on an analysis of data of 150 patients from Wuhan, China. Intensive Care Med 2020;46:846-8.

$5 \mathrm{Hu} \mathrm{H}$, Ma F, Xin Wei YF. Coronavirus fulminant myocarditis saved with glucocorticoid and human immunoglobulin. Eur Heart $J$ 2020;1307800.

6 Inciardi RM, Lupi L, Zaccone G, et al. Cardiac involvement in a patient with coronavirus disease 2019 (COVID-19). JAMA Cardiol 2020;5:819-24.

7 Argulian E, Sud K, Vogel B, et al. Right ventricular dilation in hospitalized patients with COVID-19 infection. JACC Cardiovasc Imaging 2020;13:2459-61.

8 Szekely Y, Lichter Y, Taieb P, et al. Spectrum of cardiac manifestations in COVID-19: a systematic echocardiographic study. Circulation 2020;142:342-53.

9 Mahmoud-Elsayed HM, Moody WE, Bradlow WM, et al. Echocardiographic findings in patients with COVID-19 pneumonia. Can J Cardiol 2020;36:1203-7.

10 Baycan OF, Barman HA, Atici A, et al. Evaluation of biventricular function in patients with COVID-19 using speckle tracking echocardiography. Int J Cardiovasc Imaging 2021;37:135-44.

11 Shi S, Qin M, Shen B, et al. Association of cardiac injury with mortality in hospitalized patients with COVID-19 in Wuhan, China. JAMA Cardiol 2020;5:802-8.

12 Ingram T, Augustine D, Colebourn C. COVID-19 clinical guidance, 2020. Available: https://bsecho.org/covid19 [Accessed 29 Oct 2020].

13 Lang RM, Badano LP, Mor-Avi V. Recommendations for cardiac chamber quantification by echocardiography in adults: an update from the American society of echocardiography and the European association of cardiovascular imaging. J Am Soc Echocardiogr.

14 Wang D, Hu B, Hu C, et al. Clinical characteristics of 138 hospitalized patients with 2019 novel coronavirus-infected pneumonia in Wuhan, China. JAMA 2020;323:1061-9.

15 Grasselli G, Tonetti T, Protti A, et al. Pathophysiology of COVID19-associated acute respiratory distress syndrome: a multicentre prospective observational study. Lancet Respir Med 2020;8:1201-8.

16 Zochios V, Jones N. Acute right heart syndrome in the critically ill patient. Heart Lung Vessel 2014;6:157-70. 